\title{
Tuning the Chiroptical Properties of Elongated Nano-Objects via Hierarchical Organization
}

Jie Gao, ${ }^{1}$ Wenbing $W u,{ }^{2}$ Vincent Lemaire, ${ }^{2}$ Alain Carvalho, ${ }^{2}$ Sylvain Nlate, ${ }^{1}$ Thierry Buffeteau, ${ }^{3}$ Reiko Oda, ${ }^{1}$ Yann Battie $*{ }^{4}$ Matthias Pauly $*,{ }^{2}$ Emilie Pouget ${ }^{* 1}$

${ }^{1}$ CNRS, Univ. Bordeaux, Bordeaux INP, Chimie et Biologie des Membranes et des Nanoobjets, UMR 5248, Allée St Hilaire, Bat B14, 33607 Pessac, France

${ }^{2}$ Université de Strasbourg, CNRS, Institut Charles Sadron, F-67000 Strasbourg, France ${ }^{3}$ Institut des Sciences Moléculaires (UMR5255 ISM), CNRS - Université de Bordeaux, 351 Cours de la Libération, 33405 Talence, France

${ }^{4}$ LCP-A2MC, Université de Lorraine, 1 Bd Arago, 57070 Metz, France 


\section{SI 1: Different steps of the formation of the silica helices and electrostatic Goldhelices.}

The formation of silica nanohelices has already been published several times. ${ }^{1,2}$ In brief, a 162-16 Gemini surfactant positively charged is complexed with the chiral tartrate counter-ions. The supramolecular assembly of the system in water forms twisted and helical nanoribbons (grouped under the generic name of nanohelices). By use of the $L$ - or the $D$-tartrate, the handedness is controlled (right- and left-handed for $L$ and $D$ tartrate respectively). In the present work, we use twisted ribbons with typical sizes of $108 \mathrm{~nm}$ pitch and $20 \mathrm{~nm}$ width. ${ }^{3}$ The silica transcription of such supramolecular assembly allows the formation of silica nanohelices with the same handedness and morphological control.

In order to facilitate the electrostatic interactions between the carboxylic groups of the ligands stabilizing the GNPs (tri-sodium citrate and tannic acid) and the silica surface, (3-aminopropyl) triethoxysilane (APTES) is previously used to modify the silica surface. The electrostatic GNPs grafting onto the silica helices is then performed in water at $20^{\circ} \mathrm{C}$.

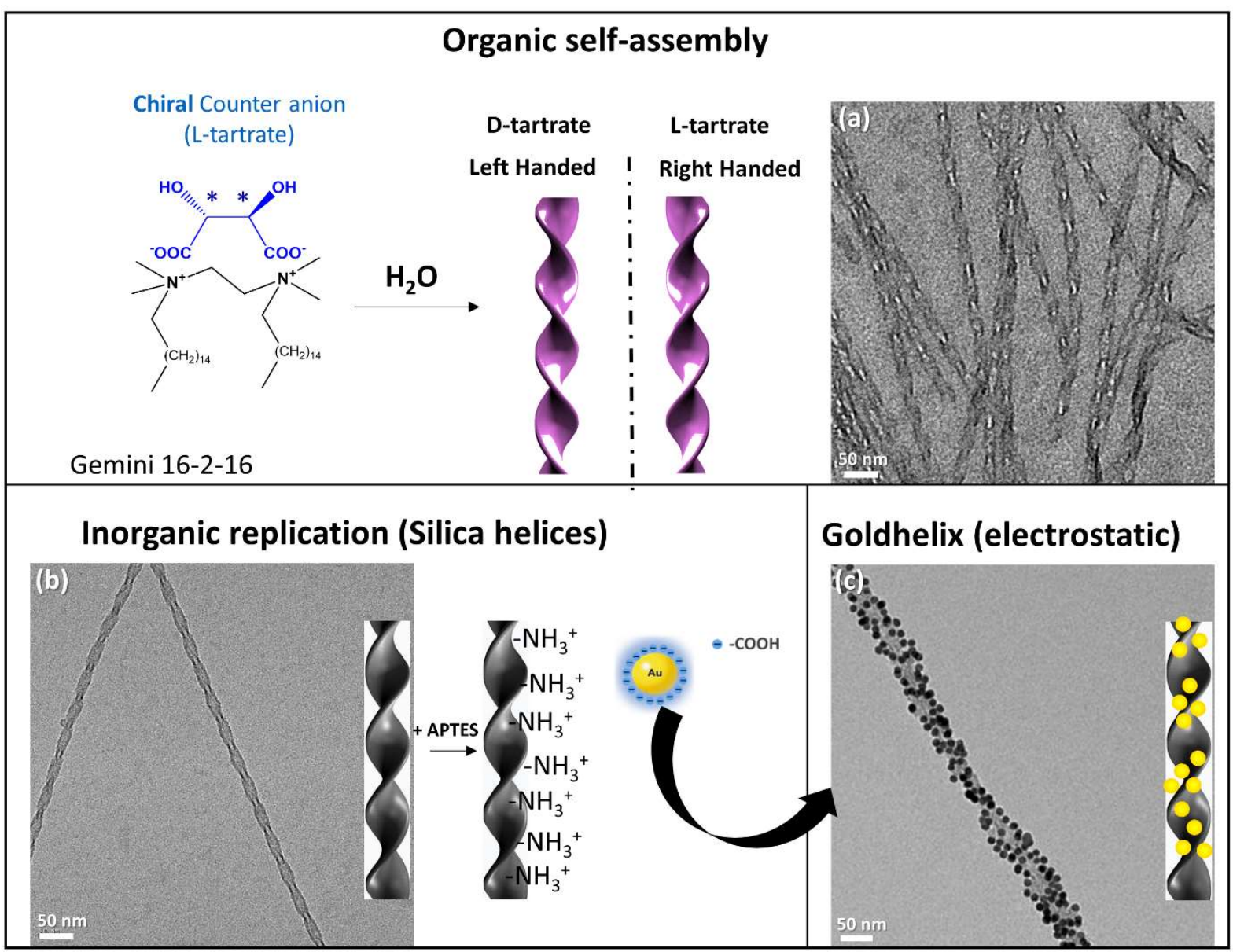

Figure SI-1: From the 16-2-16 gemini molecule to the Goldhelix. (a) Organic self-assembly. TEM image with 2\% uranyl acetate as stainer. (b) Silica replication of the organic self-assembly and (c) GNPS electrostatic grafting. 


\section{SI 2: Optimization of the GNPs covalent grafting.}

The silica structures have been modified with (3-aminopropyl) triethoxysilane (APTES) in order to enrich the surface with amine group. The GNPs are synthesized with tannic acid and tri-sodium citrate as stabilizing ligands. A ligand exchange process is then performed with $\mathrm{O}$ (2-Carboxyethyl)-O'-(2-mercaptoethyl) heptaethylene glycol ligands $\left(\mathrm{SHCH}_{2} \mathrm{CH}_{2}-\right.$ $\left.\left[\mathrm{OCH}_{2} \mathrm{CH}_{2}\right]_{8}-\mathrm{COOH}\right)$. The formation of a covalent peptide bond between the amine of the helix and the acid of the GNPs requires an initial activation of the acidic groups following wellestablished processes. ${ }^{4,5}$ 1-Ethyl-3-(3-dimethylaminopropyl)-carbodiimide (EDC) and $\mathrm{N}$ hydroxysuccinimide (NHS) are typically used to activate the - $\mathrm{COOH}$ group. Even though it is a process largely described in the literature, various doses of EDC and NHS were used in the different systems. In order to avoid the spontaneous aggregation of the GNPs during the activation process, the conditions were optimized according to the solvent nature (water or ethanol), the temperature, the pre-aging of the EDC/NHS mixture before use and the conditions of carboxylic activation.

As a first step, the $2 \mathrm{~h}$ pre-aging of the EDC/NHS mixture were tested at $4^{\circ} \mathrm{C}$ on the GNPs aggregation (before mixing with the nanohelices). Results are summarized in the following table, each condition was repeated 3 or 4 times. The "sad" symbols correspond to a non-negligible aggregation of the GNPs, the solution color turning from red to black. Such experiments reveal that the reaction in water with aged EDC/NHS solution is more suitable to prevent the GNPs aggregation.

\begin{tabular}{|c|c|c|c|c|}
\hline EDC/NHS & \multicolumn{4}{|c|}{$12 \mathrm{mM} / 60 \mathrm{mM}$} \\
\hline Solvent & \multicolumn{2}{|c|}{$\mathrm{EtOH}$} & \multicolumn{2}{|c|}{$\mathrm{H}_{2} \mathrm{O}$} \\
\hline Solution aging & Fresh solution & Aged solution & Fresh solution & Aged solution \\
\hline Results & $\Theta$ & 8 & $\Theta \odot$ & 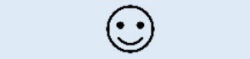 \\
\hline
\end{tabular}

In a second step, the pre-aging of the EDC/NHS solution time and the reaction temperature were optimized. The success of the reactions was evaluated by TEM and CD spectroscopy. As shown in the figure SI-2, the more suitable conditions have been evaluated to be $5 \mathrm{~h}$ of aging procedure at $4^{\circ} \mathrm{C}$.

\begin{tabular}{|c|c|c|c|c|c|c|}
\hline EDC/NHS & \multicolumn{6}{|c|}{$12 \mathrm{mM} / 60 \mathrm{mM}\left(\right.$ in $\mathrm{H}_{2} \mathrm{O}$ aged $2 \mathrm{~h}$ at $4^{\circ} \mathrm{C}$ ) } \\
\hline Reaction time & $1 \mathrm{~h}$ & $2 \mathrm{~h}$ & $3 \mathrm{~h}$ & $4 \mathrm{~h}$ & $5 \mathrm{~h}$ & $16 h$ \\
\hline $\mathrm{T}=4{ }^{\circ} \mathrm{C}$ & 8 & 8 & 8 & 8 & $\odot$ & (-) \\
\hline $\mathrm{T}=25^{\circ} \mathrm{C}$ & 8 & $\because$ & $\because$ & $\mathscr{O}$ & 8 & 8 \\
\hline
\end{tabular}

Covalent GNPs grafting time and temperature optimization. 

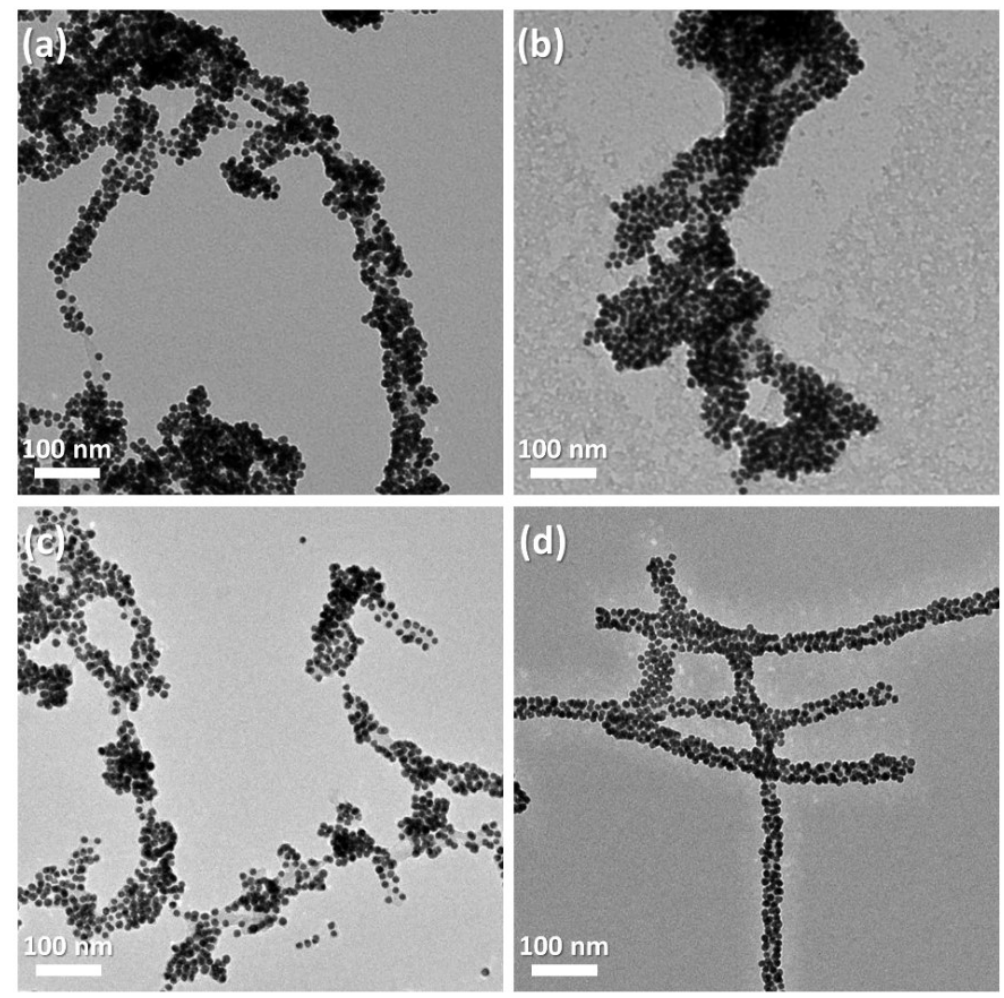

Figure SI-2: TEM images of Goldhelices obtained with pre-aging of the EDC/NHS mixture of (a) $3 \mathrm{~h}$ at $25^{\circ} \mathrm{C}$, (b) $5 \mathrm{~h}$ at $25^{\circ} \mathrm{C}$, (c) $3 \mathrm{~h}$ at $4^{\circ} \mathrm{C}$ and (d) $5 \mathrm{~h}$ at $4^{\circ} \mathrm{C}$.

The best activation conditions were the use of a $60 \mathrm{mM}$ EDC/NHS in ultra-pure water and aged $2 \mathrm{~h}$ at $4^{\circ} \mathrm{C}$ before mixing with GNPs with $-\mathrm{COOH}$ group. The optimal activation process condition is at $4^{\circ} \mathrm{C}$ at least for $5 \mathrm{~h}$. Indeed, as shown in the Figures 1 -a and SI-2-d, the GNPs cover densely and homogeneously the silica surface without any drying artifact. 


\section{SI-3: Detailed orientation analysis of the SEM images}
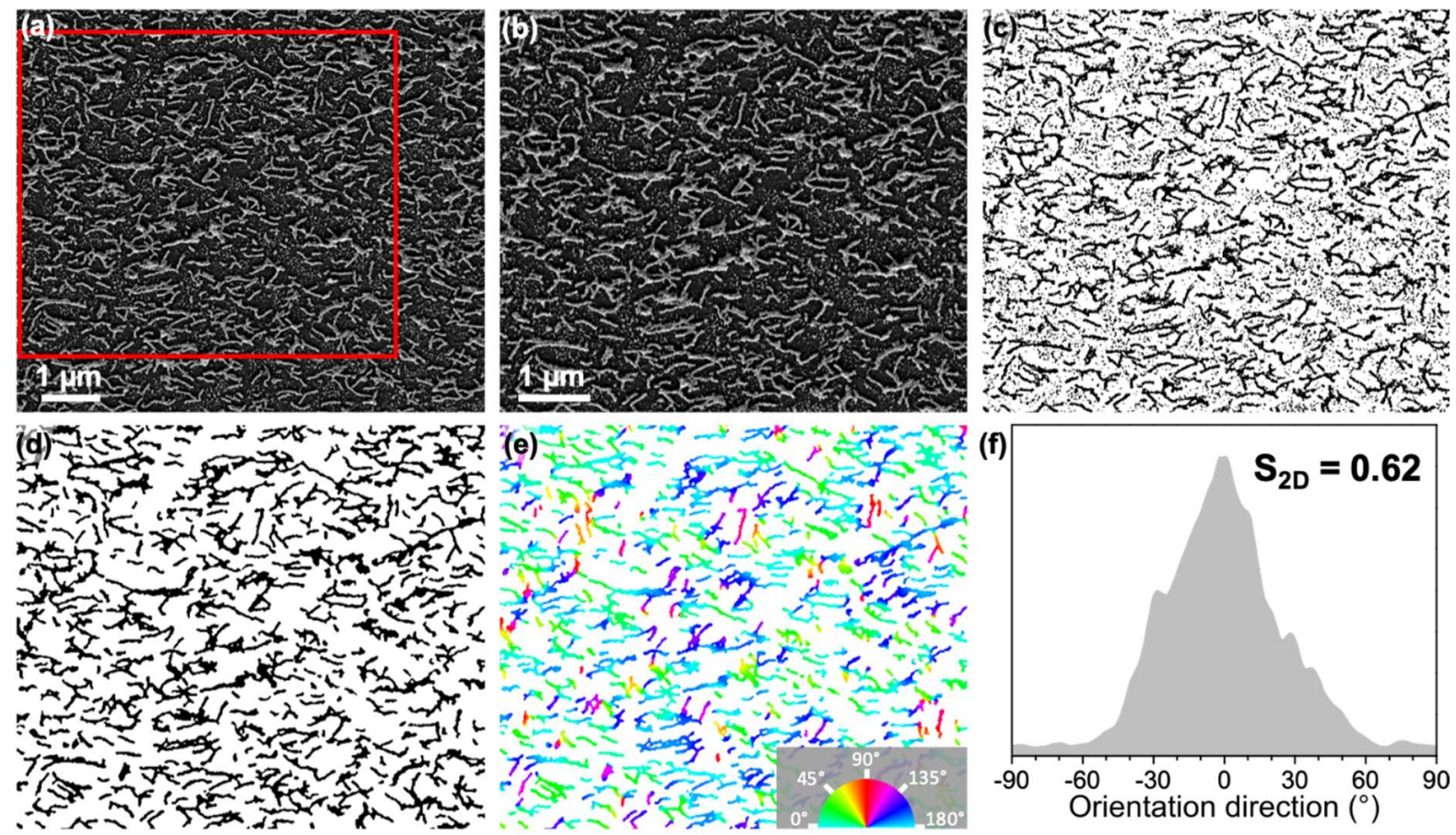

Figure S3- (a) SEM image of an oriented monolayer. (b) Selected working area (corresponding to the red rectangle in (a). (c) Binary image of (b). (d) Binary image in which the free GNPs between the Goldhelices have been removed. (e) Color-coded image depending on the orientation direction determined from the OrientationJ plugin for ImageJ (the inset shows the color code of the orientation direction). (f) Orientation distribution extracted from the image.

Starting from a SEM image (Figure S3-a), an area of interest is selected (Figure S3-b). The image is binarized using Image J (Figure S3-c), and the small spherical nanoparticles are removed from the binarized image (Figure S3-d). The density of deposited Goldhelices can be calculated as the ratio of black pixels (corresponding to the Goldhelices) to the total number of pixels of the image.

The orientation is analyzed using the plugin Orientation $J^{6}$ developed for ImageJ. It determines the local orientation of each pixel based on its neighborhood, and each pixel is colorcoded according to its orientation angle (Figure S3-e). The structure tensor is obtained with a cubic spline gradient on a 5 pixel window. The resulting image is composed with the color coding for the orientation with a constant brightness and the saturation corresponding to the original image. The distribution of orientations can be extracted from the analysis by excluding the pixels on which the coherency is less than $10 \%$, and centered around its maximum (Figure S3-f).

Finally, the $2 \mathrm{D}$ order parameter is defined as $\mathrm{S}_{2 \mathrm{D}}=<2 \cos ^{2} \delta-1>$, where $\delta$ is the angle between the direction attributed to each pixel and the main orientation direction. The nematic order parameter is calculated as the average of " $2 \cos ^{2} \delta$ - 1 " weighted by the population for each angle $\delta$. 


\section{SI-4: CDM modelization of the Goldhelices}

The optical properties of Goldhelices are simulated by using the coupled dipole method (CDM). CDM takes into account the dipolar interaction between nanoparticles in the same helix. Each spherical nanoparticle, located at a position $\overrightarrow{r_{m}}$ is considered as a point dipole which has a polarizability:

$\alpha_{m}=R_{m}^{3} \frac{\varepsilon_{n p}\left(R_{m}\right)-\varepsilon_{h}}{\varepsilon_{n p}\left(R_{m}\right)+2 \varepsilon_{h}}$.

$\varepsilon_{h}=2.1$ is the dielectric constant of the $\mathrm{SHCH}_{2} \mathrm{CH}_{2}-\left[\mathrm{OCH}_{2} \mathrm{CH}_{2}\right]_{8}-\mathrm{COOH}$ molecular coating. $R_{m}$ and $\varepsilon_{n p}\left(R_{m}\right)$ are the radius and the dielectric function of the $\mathrm{m}^{\text {th }}$ nanoparticle. This latter is calculated according to the Drude model:

$\varepsilon_{n p}\left(R_{m}\right)=\varepsilon_{n p}(\infty)-\frac{\omega_{p}^{2}}{\omega\left(\omega+j \Gamma_{0}\right)}+\frac{\omega_{p}^{2}}{\omega\left(\omega+j\left(\Gamma_{0}+\frac{v_{f}}{R_{m}}\right)\right)}$.

$\varepsilon_{n p}(\infty)$ is the dielectric function of gold given by Palik, ${ }^{7}$ while $\omega_{p}=8.8 \mathrm{eV}, v_{f}=1.4 \times 10^{6} \mathrm{~m} \cdot \mathrm{s}^{-1}$ and $\Gamma_{0}=0.081 \mathrm{eV}$ are the plasma energy, the Fermi velocity and the damping of free electrons of gold, respectively. The local field $\overrightarrow{E_{l o c, m}}$ view by the $\mathrm{m}^{\text {th }}$ nanoparticle induces a dipole moment $\overrightarrow{P_{m}}$ expressed as:

$\overrightarrow{P_{m}}=\alpha_{m} \overrightarrow{E_{l o c, m}}$

The local field $\overrightarrow{E_{l o c, m}}$ is the sum of the incident electric field $\overrightarrow{E_{0, m}}$ and the field scattered by the other nanoparticles 1 :

$\alpha_{m}^{-1} \overrightarrow{P_{m}}=\overrightarrow{E_{0, m}}-\sum_{l \neq m}^{N} A_{m l} \overrightarrow{P_{l}}$,

where $\mathrm{N}$ is the number of nanoparticles in the structure. The second term of equation (4), which describes the electromagnetic interactions between nanoparticles, is given by

$A_{m l} \overrightarrow{P_{l}}=\frac{e^{j k r_{m l}}}{r_{m l}^{3}}\left\{k^{2} \overrightarrow{r_{m l}} \wedge \overrightarrow{r_{m l}} \wedge \overrightarrow{P_{l}}+\frac{1-j k r_{m l}}{r_{m l}^{2}}\left(r_{m l}^{2} \overrightarrow{P_{l}}-3 \overrightarrow{r_{m l}} \cdot\left(\overrightarrow{r_{m l}} \cdot \overrightarrow{P_{l}}\right)\right)\right\}$

where $\mathrm{k}$ is the norm of wave vector $\vec{k}$. This latter is normal to the Goldhelix axis. $\overrightarrow{r_{m l}}=\overrightarrow{r_{m}}-$ $\overrightarrow{r_{l}}$. By using the same procedure for all nanoparticles, we generate a set of $3 \mathrm{~N}$ linear equations which can be rewritten in a more compact form:

$E_{0}=A P$

$P$ and $E_{0}$ are the $3 \mathrm{~N}$ dipole moment and incident electric field vectors. The tensor $\mathrm{A}$ is given by combining equations 4 and 5 . The vector $P$ is obtained by solving equation 6 by using the generalized minimal residual method (GMRES). The extinction cross section and the phase-lag cross section of the structure are then calculated for a given polarization by using the following equations: 
$\sigma=\frac{4 \pi}{\left|E_{0}\right|^{2}} \sum_{m=1}^{N} \operatorname{Im}\left(\overrightarrow{E_{0, m}} \cdot \overrightarrow{P_{m}}\right)$,

$\varphi=\frac{2 \pi k}{\left|E_{0}\right|^{2}} \sum_{m=1}^{N} \operatorname{Re}\left({\overrightarrow{E_{0, m}}}^{*} \cdot \overrightarrow{P_{m}}\right)$,

In this simulation, we consider that the nanoparticles are located in a homogeneous silica matrix, i.e. we neglect the interface between the silica helix and air where the nanoparticles are located. We also neglect the interaction between the nanoparticles located on different helices. In addition, we assume that nanoparticles are monodispersed in size $\left(R_{m}=5 \mathrm{~nm}\right)$ and are perfectly organized. The positions of nanoparticles are calculated as following. First, the nanoparticles are perfectly organized, on a flat ribbon, into a two dimensional square lattice. The interparticle distance is $14 \mathrm{~nm}$. Then, the ribbon is twisted with the geometrical parameters estimated by TEM (108 $\mathrm{nm}$ in pitch and $20 \mathrm{~nm}$ in width). The length of the Goldhelix is 520 nm.

\section{SI-5: Transmittance spectra of a single layer of Goldhelix}

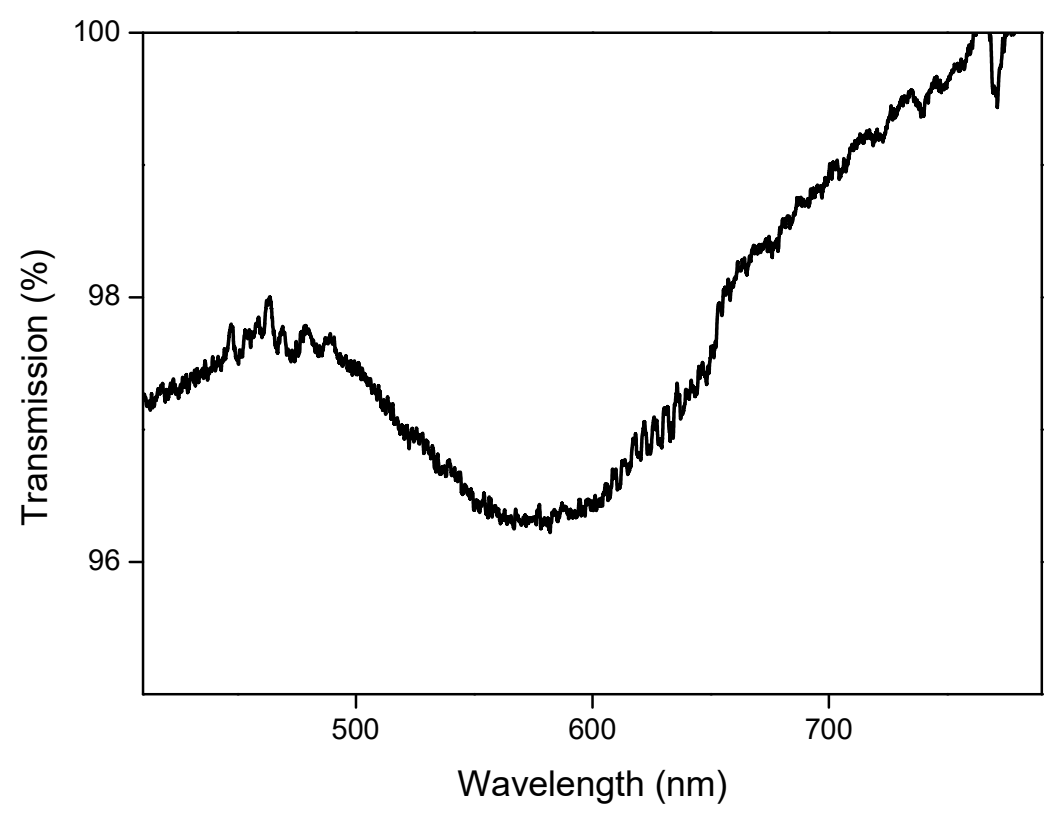




\section{SI-6: Number of Goldhelix layers to create perfect polarizers}

\section{Oriented sample: formation of linear polarizer}

The Mueller matrix measured for 1 layer $M_{1}$ can be defined from the differential decomposition formalism:

$M_{1}=\exp \left(L_{1}\right),(9)$

with $\mathrm{L}_{1}$ the matrix in which appears the terms LD, LD ', LB LB', CD and CB. For an oriented sample, the LB is negligible when the LD reach its maximum value. At the corresponding wavelength, the matrix $L_{1}$ is thus of the form:

$L_{1}=\left(\begin{array}{llll}0 & a & 0 & 0 \\ a & 0 & 0 & 0 \\ 0 & 0 & 0 & 0 \\ 0 & 0 & 0 & 0\end{array}\right)$.

Here, it has been assumed that the CD is negligible with respect to LD. Moreover, we considered the azimuth along the helices main axis. The Mueller matrix of a sample with $\mathrm{N}$ layers of the same thickness is given by:

$M_{N}=\exp \left(\mathrm{N} L_{1}\right) \cdot(11)$

$\mathrm{M}_{\mathrm{N}}$ can be rewritten as following:

$M_{N}=\left(\begin{array}{cccc}1 & \tanh (N a) & 0 & 0 \\ \tanh (N a) & 1 & 0 & 0 \\ 0 & 0 & 1 / \cosh (N a) & 0 \\ 0 & 0 & 0 & 1 / \cosh (N a)\end{array}\right)$

A perfect linear polarizer has a Mueller matrix of the form:

$M_{\text {lin }}=\left(\begin{array}{cccc}1 & \pm 1 & 0 & 0 \\ \pm 1 & 1 & 0 & 0 \\ 0 & 0 & 0 & 0 \\ 0 & 0 & 0 & 0\end{array}\right)$

We can demonstrate that $M_{N}$ can be assimilated to $M_{\text {lin }}$ for an infinite number of layer. Figure SI-6-a shows the simulated $\mathrm{m}_{12}=\mathrm{m}_{21}$ and $\mathrm{m}_{33}=\mathrm{m}_{44}$ element of the $\mathrm{M}_{\mathrm{N}}$ matrix. 


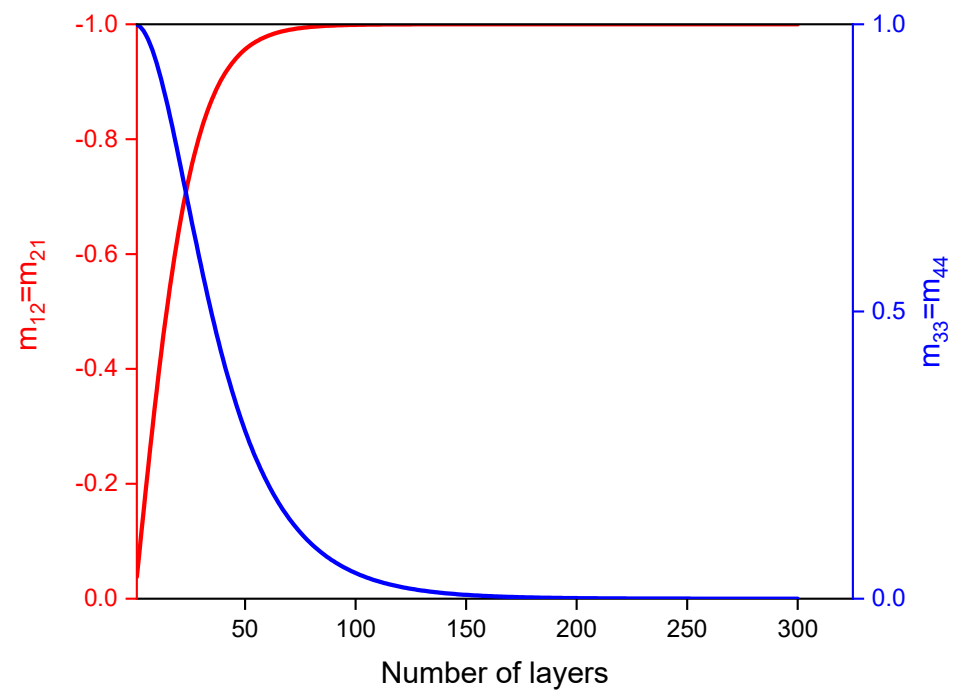

Graph SI-6-a-Evolution of the $m_{12}=m_{21}$ and $m_{33}=m_{44}$ terms regarding the number of Goldhelix layers.

From 100 layers, the sample tends to be a perfect linear polarizer. Indeed, regardless of the input polarization state, the output polarization will be linear perpendicular to the axis of the helices.

\section{Non-oriented sample: formation of circular polarizer}

The $\mathrm{L}_{1}$ matrix of non-oriented GoldHelix film has the following form:

$L_{1}=\left(\begin{array}{llll}0 & 0 & 0 & a \\ 0 & 0 & 0 & 0 \\ 0 & 0 & 0 & 0 \\ a & 0 & 0 & 0\end{array}\right)$

Here, we neglect the CB. The Mueller matrix $M_{N}$ of a sample with $\mathrm{N}$ layers of the same thickness is given by:

$M_{N}=\exp \left(\mathrm{N} L_{1}\right) \cdot(15)$

After simplifications, $\mathrm{M}_{\mathrm{N}}$ can be rewritten as following:

$$
M_{N}=\left(\begin{array}{cccc}
1 & 0 & 0 & \tanh (N a) \\
0 & 1 / \cosh (N a) & 0 & 0 \\
0 & 0 & 1 / \cosh (N a) & 0 \\
\tanh (N a) & 0 & 0 & 1
\end{array}\right)
$$

A perfect circular polarizer has a Mueller matrix of the form: 
$M_{\text {cir }}=\left(\begin{array}{cccc}1 & 0 & 0 & \pm 1 \\ 0 & 0 & 0 & 0 \\ 0 & 0 & 0 & 0 \\ \pm 1 & 0 & 0 & 1\end{array}\right)$

We can demonstrate that $M_{N}$ can be assimilated to $M_{c i r}$, for an infinite number of layer.

Figure SI-6-b shows the simulated $\mathrm{m}_{41}=\mathrm{m}_{14}$ element of the $\mathrm{M}_{\mathrm{N}}$ matrix.

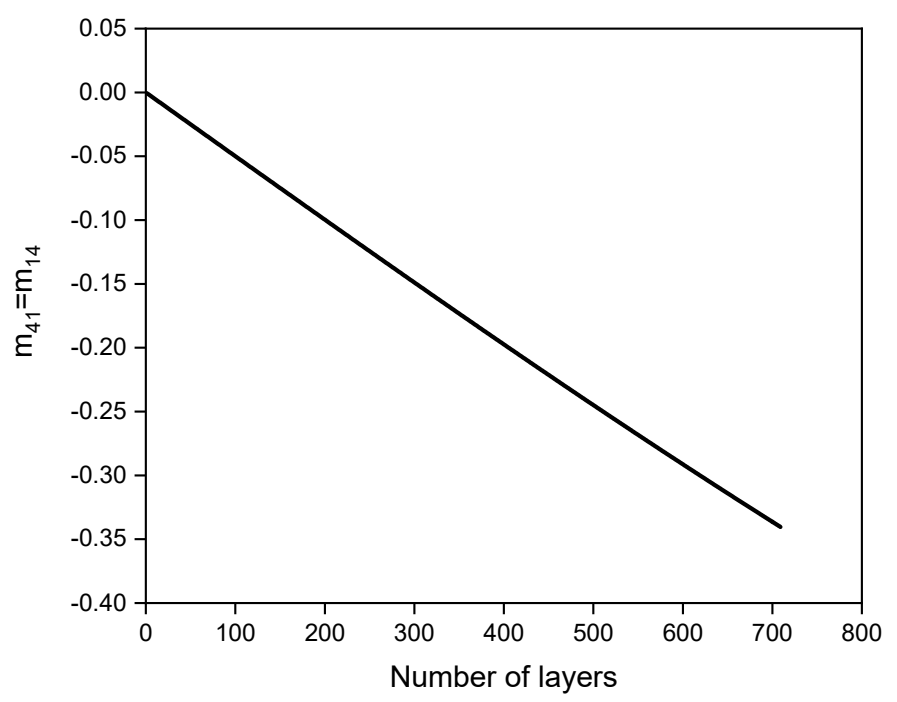

Graph SI-6-b-Evolution of the CD term of the Mueller matrix $m 41$ regarding the number of Goldhelix layers.

The parameter $\mathrm{m}_{41}=\mathrm{m}_{14}$ directly gives the degree of circular polarization. The closer it is to 1 (in absolute value) the more the transmitted light will be circularly polarized. The graph SI-6$\mathrm{b}$ shows that the number of layers should be very high to tend to a perfect circular polarizer. 


\section{REFERENCES}

${ }^{1}$ Delclos, T.; Aimé, C.; Pouget, E.; Brizard, A.; Huc, I.; Delville, M.-H.; Oda, R. Individualized Silica Nanohelices and Nanotubes: Tuning Inorganic Nanostructures Using Lipidic Self-Assemblies. Nano Lett. 2008, 8, 1929-1935.

${ }^{2}$ Okazaki, Y.; Cheng, J.; Dedovets, D.; Kemper, G.; Delville, M.-H.; Durrieu, M.-C.; Ihara, H.; Takafuji, M.; Pouget, E.; Oda, R. Chiral Colloids: Homogeneous Suspension of Individualized $\mathrm{SiO}_{2} \mathrm{Helical}_{\text {and }}$ Twisted Nanoribbons. ACS Nano 2014, 8, 6863-6872.

${ }^{3}$ Oda, R; Huc, I.; Schmutz, M.; Candau, S. J.; MacKintoshet, F. C. Tuning Bilayer Twist Using Chiral Counterions. Nature 1999, 399, 566-569.

4. Wang, C.; Yan, Q.; Liu, H.-B.; Zhou, X.-H.; Xiao, S.-J. Different EDC/NHS Activation Mechanisms between PAA and PMAA Brushes and the Following Amidation Reactions. Langmuir 2011, 27, 12058-12068.

5. Sam, S.; Touahi, L. Salvador Andresa, J.; Allongue, P.; Chazalviel, J.-N.; Gouget-Laemmel, A. C.; Henry de Villeneuve, C.; Moraillon, A.; Ozanam, F.; Gabouze, N.; Djebbar, S. Semiquantitative Study of the EDC/NHS Activation of Acid Terminal Groups at Modified Porous Silicon Surfaces, Langmuir 2010, 26, 809-814.

${ }^{6}$ Rezakhaniha, R.; Agianniotis, A. ; Schrauwen, J. T. C. ; Griffa, A. ; Sage, D. ; Bouten, C. V. C. ; Vosse, F. N. ; Unser M. ; Stergiopulos, N. Experimental Investigation of Collagen Waviness and Orientation in the Arterial Adventitia Using Confocal Laser Scanning Microscopy. Biomech. Model. Mechanobiol. 2012, 11, 461-473.

7. Palik, E. D. Handbook of Optical Constants of Solids, $1^{\text {st }}$ edition; Academic Press: Boston, 1998. 